\title{
Early forest fire detection using Low Energy Hydrogen Sensors
}

\author{
$\underline{\text { Kai Nörthemann }}^{1 \text { a }}$, Jan-Eric Bienge ${ }^{2}$, Michael Dallmer $^{1}$, Jürgen Müller ${ }^{2}$, Michael Milstrey $^{3}$, Michael $^{1}$ \\ Rothe $^{4}$, Werner Moritz \\ ${ }^{1}$ Humboldt-Universität zu Berlin, Brook-Taylor-Str. 2, 12489 Berlin, Germany \\ ${ }^{2}$ Johann Heinrich von Thünen-Institut, Alfred-Möller-Straße 1, 16225 Eberswalde, Germany \\ ${ }^{3}$ Swissbit Germany AG, Wolfener Str. 36, 12681 Berlin, Germany \\ ${ }^{4}$ DResearch Digital Media Systems GmbH, Otto-Schmirgal-Str. 3, 10319 Berlin, Germany \\ akai.noerthemann@hu-berlin.de
}

\begin{abstract}
:
Most of the huge forest fires starts in partial combustion. In the beginning of smouldering fire emission of hydrogen in low concentration occurs. Therefore, hydrogen can be used to detect forest fires before open flames are visible and high temperatures are generated.

The hydrogen sensor which has been developed is a metal insulator semiconductor (MIS) structure which allows an economical production. Due to the low energy consumption an autarkic working unit in the forest was established. In this contribution first experiments are shown demonstrating the possibility to detect forest fires at a very early stage using the hydrogen sensor.
\end{abstract}

Key words: hydrogen sensor, low concentration, forest fire, low energy consumption

\section{Introduction}

Every year a lot of forest fires occur in the world. During this burning a huge area is destroyed and human settlements have to be rebuild. So there is an economical interest to detect the fire as soon as possible to reduce the damage.

Present forest fire detection systems are based on the detection of effects, which appear when an open fire already exists. Most of the systems determine heat or dust particles. Not so common is the detection of gases which occur by smouldering of organic matter before an open fire accrues. In experiments the detector determines the concentration of hydrogen formed by a smouldering fire. The concentration of created hydrogen in such an environment is about $20 \mathrm{ppm}$. For the outdoor application a low energy setup was developed. Here we present first results of field experiments in scots pine stands. Using wood and grass a smouldering fire was created in the forest. The sensors where placed at different distances from the fire place, to monitor the sensor signals.

\section{Experiments}

The sensor is a metal insulator semiconductor (MIS) structure. An explicit sketch is shown in figure 1. Silicon oxide and silicon nitride insulators are grown on a silicon waver.
Afterward a lanthanum trifluoride layer is grow by physical vapour deposition at high vacuum. On top a palladium gate metal layer is produced by DC sputtering.

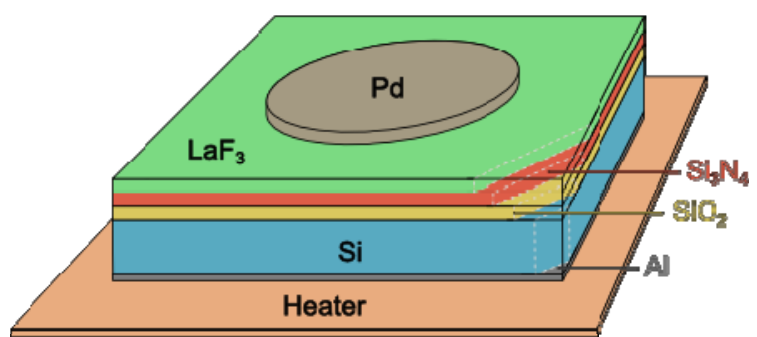

Fig. 1. Sketch of the capacitive sensor structure mounted on heater.

These stack is a capacitive element and the capacitance depends on the voltage between gate (palladium) and bulk (silicon). Details of this hydrogen sensor is described else where. [1] Between the backside aluminum contact and the gate the capacitance is measured. These structure is bonded on top of a ceramic heater which is used for the activation of the sensor. The sensors where activated once a day to improve the response time and the signal. During the operation the sensor has not to be heated. The measurements can be done at ambient temperature. Due to this fact the power consumption of the electronic is low, and operating with batteries is capable. 


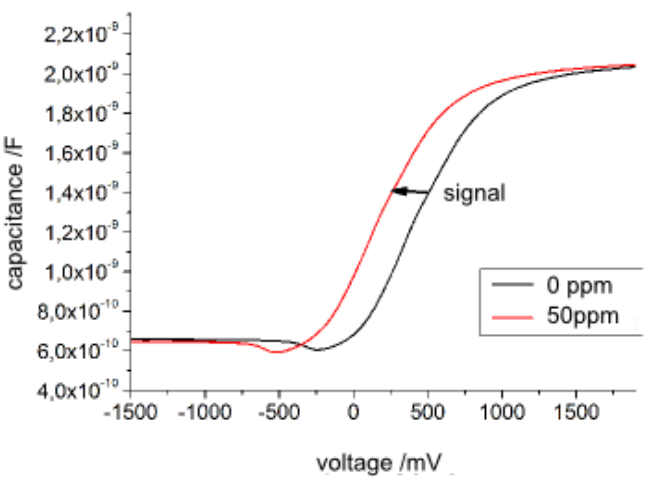

Fig. 2. Change of the Capacitance Voltage plot due to hydrogen uptake. When hydrogen occurs at the palladium surface the graph is shifted to lower potential.

When hydrogen reacts with the sensor structure the capacitance/voltage behavior is changing, which is shown in figure 2.

The hydrogen molecule dissociates at the palladium surface. The hydrogen atom is solved in the palladium and interacts with the lanthanum trifluoride layer. By this, additional charges occur and the capacitance voltage plot is shifted to lower potential in comparison to absence of hydrogen. The potential of constant capacitance is proportional to the logarithm of the concentration. To determine the hydrogen concentration only the voltage has to be measured. For the field experiment an electronic design was developed which selfactuating changes the voltage to keep the capacitance constant. The electronic allows to store the measured values and send the data via a RF interface to a PC. This PC collects the data from several sensors. With this wireless communication it is possible to arrange the sensors at different positions in a large area in the forest.

\section{Results}

The experimental environment was established inside of a mixed forest. For safety reasons the smouldering fire was started in a metal bowl. Around the fire place the sensors where mounted at different trees. During the measurements the height of the sensors where changed between $1 \mathrm{~m}$ and $3 \mathrm{~m}$. And the horizontal distance from fire to sensor was between $10 \mathrm{~m}$ and $80 \mathrm{~m}$. In figure 3 the sensor signals in a distance of $10 \mathrm{~m}$ are compared at the height of $1 \mathrm{~m}$ and $3 \mathrm{~m}$. It is evident that the height of the sensor has an influence on the signal. Due to the wind and crown the major effect was measured at a height of $1 \mathrm{~m}$ at this experiment. Additionally the influence of the wind direction becomes apparent. Not all sensors detect hydrogen due to the wind direction.

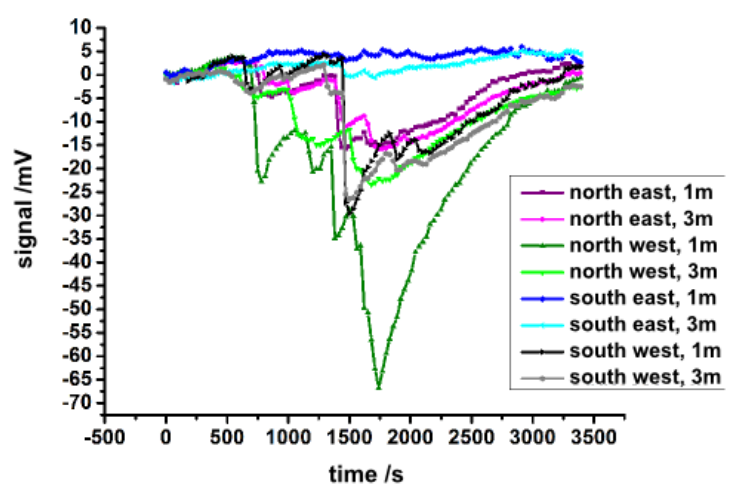

Fig. 3. Experiment in the forest with distance between fire and sensors of $10 \mathrm{~m}$. The sensors were placed in four directions at a height of $1 \mathrm{~m}$ and $3 \mathrm{~m}$. The dependency of the wind direction is visible. Also the influence of the detector height can be observed. In this configuration the main signal was observed at a height of $1 \mathrm{~m}$

At larger distances the hydrogen is still detectable. In the plot the base line is not flat. This effect is caused by a not optimized sensor shielding. Up to now environment effects such as light incidence, temperature and moisture change are not completely eliminated. Figure 4 shows data points in an interspacing of $50 \mathrm{~m}$. A significant change in the potential is visible.

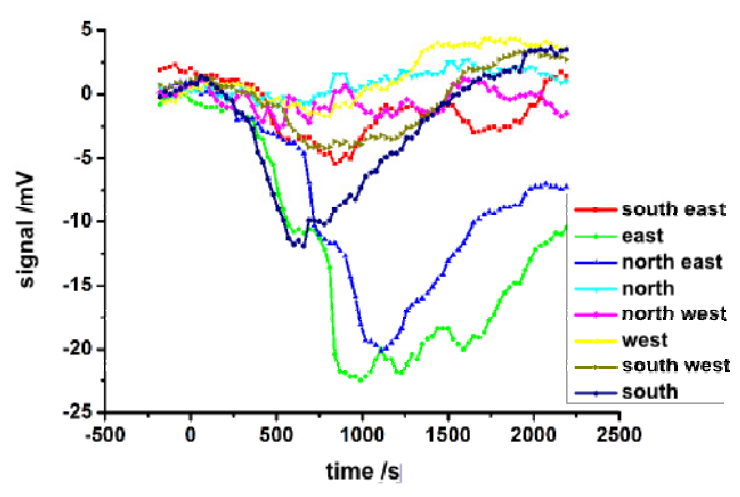

Fig. 4. Experiment in the forest with a distance between fire and sensors of $50 \mathrm{~m}$. The sensors were mounted at a height of $3 \mathrm{~m}$.

\section{Discussion and Outlook}

The measurements have shown that with increasing wind speed it becomes easier to detect hydrogen at distant positions. High wind speed is one of the factors favouring forest fires.

In these first experiments we have shown that hydrogen is formed during smouldering fire and these hydrogen is detectable outside in the forest with the new developed sensors. The 
shielding of the detectors has to be optimized to avoid the influence of light incidence, moisture change and temperature differences. The response time was below five minutes from the time the fire was started. This time includes the convection of the hydrogen from the fire to the sensor over a distance up to $50 \mathrm{~m}$. When many detectors are distributed over the forest it is possible to correlate the different signals. By using an intelligent algorithm it is possible to determine the correct position of the fire to avoid false alarms.

\section{Acknowledgements}

This work was financial supported by Zentrales Innovationsprogramm Mittelstand (ZIM) in the project InPriWa,

\section{References}

[1] W. Moritz, V. Fillipov, A. Vasiliev, G.

Cherkashinin, J.Szeponik, ECS Transactions, 3

(10) 223-230 (2006); doi: 10.1149/1.2357262 